LAWRENCE LIVERMORE NATIONAL LABORATORY

\section{On the Fielding of a High Gain, Shock-Ignited Target on the National Ignition Facility in the Near Term}

L. J. Perkins, R. Betti, G. P. Schurtz, R. S. Craxton, A. M. Dunne, A. J. Mackinnon, K. N. LaFortune, A. J. Schmitt, P. W. McKenty,D. S. Bailey, M. A. Lambert, X. Ribeyre, W. R. Theobald, D. J. Strozzi, D. R. Harding, A. Casner, G. V. Erbert, K. S. Andersen, M. Murakami, A. J. Comley, S. Atzeni, R. C. Cook, R. B. Stephens

Revision 0

April 23, 2010 


\section{Disclaimer}

This document was prepared as an account of work sponsored by an agency of the United States government. Neither the United States government nor Lawrence Livermore National Security, LLC, nor any of their employees makes any warranty, expressed or implied, or assumes any legal liability or responsibility for the accuracy, completeness, or usefulness of any information, apparatus, product, or process disclosed, or represents that its use would not infringe privately owned rights. Reference herein to any specific commercial product, process, or service by trade name, trademark, manufacturer, or otherwise does not necessarily constitute or imply its endorsement, recommendation, or favoring by the United States government or Lawrence Livermore National Security, LLC. The views and opinions of authors expressed herein do not necessarily state or reflect those of the United States government or Lawrence Livermore National Security, LLC, and shall not be used for advertising or product endorsement purposes.

\section{Auspices Statement}

This work performed under the auspices of the U.S. Department of Energy by Lawrence Livermore National Laboratory under Contract DE-AC52-07NA27344. 


\title{
On the Fielding of a High Gain, Shock-Ignited Target on the National Ignition Facility in the Near Term
}

\author{
L. J. Perkins ${ }^{1}$, R. Betti ${ }^{2}$, G. P. Schurtz ${ }^{3}$, R. S. Craxton ${ }^{2}$, A. M. Dunne ${ }^{4}$, A. J. Mackinnon ${ }^{1}$, \\ K. N. LaFortune ${ }^{1}$, A. J. Schmitt ${ }^{5}$, P. W. McKenty ${ }^{2}$, D. S. Bailey ${ }^{1}$, M. A. Lambert ${ }^{1}$, \\ X. Ribeyre ${ }^{3}$, W. R. Theobald ${ }^{2}$, D. J. Strozzi ${ }^{1}$, D. R. Harding ${ }^{2}$, A. Casner ${ }^{6}$, G. V. Erbert ${ }^{1}$, \\ K. S. Andersen ${ }^{2}$, M. Murakami ${ }^{7}$, A. J. Comley ${ }^{8}$, S. Atzeni ${ }^{9}$, R. C. Cook $^{1}$, R. B. Stephens ${ }^{10}$ \\ ${ }^{1}$ Lawrence Livermore National Laboratory, Livermore CA, USA \\ ${ }^{2}$ Laboratory for Laser Energetics, University of Rochester, Rochester NY, USA \\ ${ }^{3}$ Centre Lasers Intenses et Applications, University of Bordeaux, France \\ ${ }^{4}$ Central Laser Facility, Rutherford Appleton Laboratory, UK \\ ${ }^{5}$ Naval Research Laboratory, Washington DC, USA \\ ${ }^{6}$ CEA, DAM, DIF, Arpajon, France \\ ${ }^{7}$ Institute of Laser Engineering, Osaka University, Osaka, Japan \\ ${ }^{8}$ Centre for Inertial Fusion Studies, Imperial College London, UK \\ ${ }^{9}$ Dipartimento di Energetica, University of Rome La Sapienza, Rome Italy \\ ${ }^{10}$ General Atomics, San Diego CA, USA
}

\begin{abstract}
Shock ignition, a new concept for igniting thermonuclear fuel, offers the possibility for a near-term ( 3-4 years) test of high gain inertial confinement fusion on the National Ignition Facility at less than 1MJ drive energy and without the need for new laser hardware. In shock ignition, compressed fusion fuel is separately ignited by a strong spherically converging shock and, because capsule implosion velocities are significantly lower than those required for conventional hotpot ignition, fusion energy gains of $\sim 60$ may be achievable on NIF at laser drive energies around $\sim 0.5 \mathrm{MJ}$. Because of the simple all-DT target design, its in-flight robustness, the potential need for only 1D SSD beam smoothing, minimal early time LPI preheat, and use of present (indirect drive) laser hardware, this target may be easier to field on NIF than a conventional (polar) direct drive hotspot ignition target. Like fast ignition, shock ignition has the potential for high fusion yields at low drive energy, but requires only a single laser with less demanding timing and spatial focusing requirements. Of course, conventional symmetry and stability constraints still apply. In this paper we present initial target performance simulations, delineate the critical issues and describe the immediate-term R\&D program that must be performed in order to test the potential of a high gain shock ignition target on NIF in the near term.
\end{abstract}

\section{The Potential of Shock Ignition on NIF}

The principle of shock ignition [1] is shown in Fig.1. Here we illustrate schematically the laser pulse shape required to drive a conventional NIF hotspot ignition target (dotted curve) in comparison with that for a prospective shock ignition target (solid curve). In the conventional target whether direct or indirect drive, the laser driver pulse is 
required to assemble the fuel at high density and impart a sufficiently high velocity $\left(\sim 3.5-4 \times 10^{7} \mathrm{~cm} / \mathrm{s}\right)$ to the imploding shell so that its $P d V$ work creates the central ignition hotspot on stagnation [2]. In this regard, conventional direct- or indirect-drive hotspot ignition could be designated as occurring through "fast-compression".

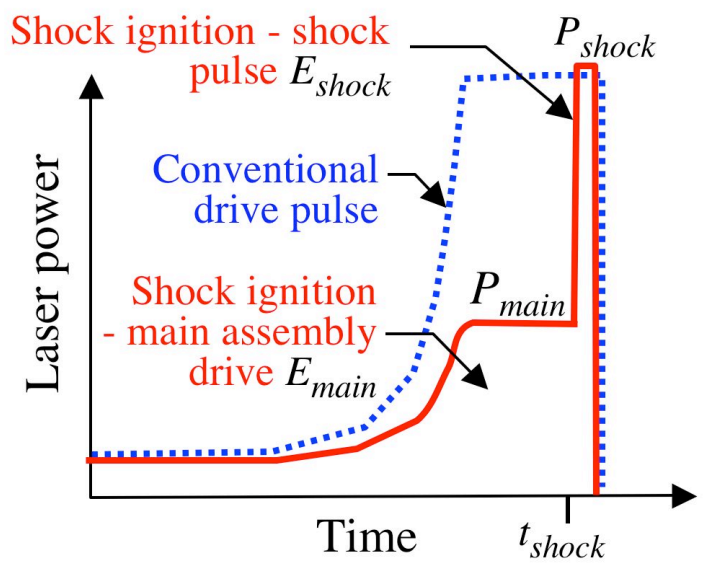

FIG. 1. Schematic laser pulse shape for shock ignition (solid curve) relative to that for conventional indirect or direct drive hotspot ignition (dotted curve). A near-term NIF shock ignited target at $\sim 0.5 \mathrm{MJ}$ drive energy would require a main drive power of $P_{\text {main }} \sim 95 T W$ and a shock drive power of $P_{\text {shock }} \leq 350 T W$ (see below)

By contrast, in shock ignition [1], the fuel assembly and ignition phases are decoupled as follows: The cryogenic shell is initially imploded on a low adiabat using a laser drive of modest peak power and lower total energy. While the resulting low implosion velocity yields only a low temperature central region, the low adiabat of the fuel leads to high values of the areal and mass densities. The assembled fuel is then separately ignited from a central hotspot heated by a strong, spherically-convergent shock driven by the high intensity spike at the end of the laser pulse. The launching of the ignition shock is timed to reach the center just as the main fuel is stagnating and starting to rebound. For larger, high yield shock ignited NIF targets at greater than 1MJ total drive energy, the majority of the laser energy is contained in the main portion of the pulse required for initial fuel compression, while only a modest energy fraction $(\sim 25 \%)$ is required for the shock ignition [3]. For smaller, sub-MJ targets, optimum energy partitioning is closer to 50:50 (see below). Because the implosion velocity is significantly less than required for conventional (fast-compression) hotspot ignition on NIF, considerably more fuel mass can be assembled for the same kinetic energy in the shell, 


\section{LLNL Technical Report LLNL-TR-428513, Revision O - April 23, 2010}

potentially offering significantly higher fusion gains/yields for the same laser energy or, equivalently, retaining acceptable gains at appreciably lower drive energies.

We have performed an initial study exploring the scaling of $1 \mathrm{D}$ energy gains for candidate shock-ignited target designs on NIF [3]. The results are shown in Fig. 2 where the fusion energy yields and gain curve (blue points) are plotted as a function of the total delivered laser energy (i.e., the sum of the main assembly and shock laser energy) and were obtained from 1D LASNEX simulations. For comparison, we show the present predicted performance of the NIF ignition baseline target $(\mathrm{CH}$ ablator) under conventional indirect drive (green point) together with 2D gain predictions from two studies of NIF target designs operating under conventional symmetric direct drive (DD) and polar direct drive (PDD) at $1 \mathrm{MJ}$ drive energy $[4,5,6,7]$. The yield and gain curves in Fig 2 were obtained for NIF shock ignition targets with wetted $\mathrm{CH}$ foam ablators. We also indicate the performance for a candidate near term, shock ignited target for NIF (red point) based on a simple all-DT (fuel+ablator) configuration; as discussed below, this offers the potential for (1D) gains of $\sim 60$ ( $\sim 30 \mathrm{MJ}$ yield) at $\sim-0.5 \mathrm{MJ}$ drive energy .

(a)

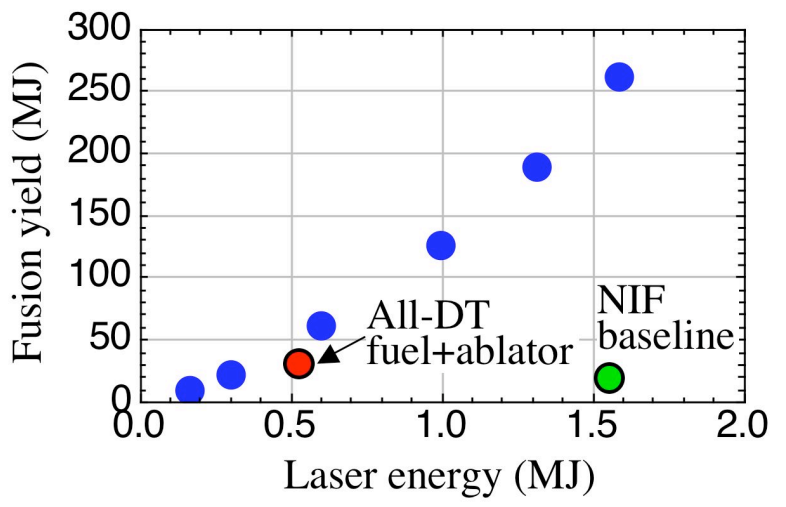

(b)

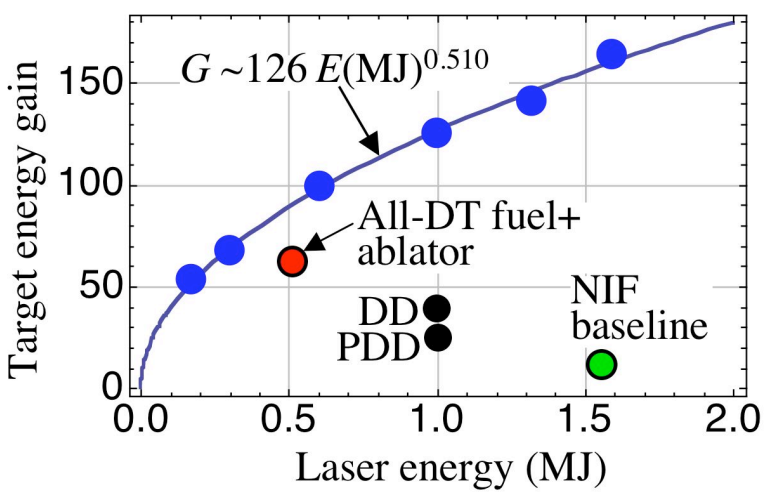

Fig.2. (a) Fusion yield curve, (b) target energy gain curve, versus total laser drive energy for NIF shock ignited targets (wetted foam ablators) from ID LASNEX simulations [3]. Corresponding performance for the NIF indirect drive baseline ignition target with $\mathrm{CH}$ ablator (green point) is shown for comparison, together with $2 D$ gain predictions for NIF targets operating under conventional symmetric direct drive (DD) and polar direct drive (PDD). Also shown is althe $1 D$ performance of a candidate near-term, gain-60 all-DT shock ignited target at $\sim 0.5 M J$ drive energy with $\sim 30 M J$ fusion yield (red point)

We caution that although the shock ignition results in Fig.2 indicate the promise for high gain at low drive energies, these were obtained in hydro-equivalent 1D simulations only. As discussed below in this paper, considerable 2D and 3D simulations, 


\section{LLNL Technical Report LLNL-TR-428513, Revision O - April 23, 2010}

plus supporting experimental $R \& D$, remains to be done to fully qualify such designs for NIF.

High gains and yields may also be attainable with "fast ignition", an alternative method of igniting ICF targets presently under study [8,9,10,11]. Fast ignition, like shock ignition, also decouples fuel assembly from the ignition process, and consequently has similar potential advantages in fusion gains/yields and lower threshold for drive energies. Fast ignition requires two physically distinct, time-synchronized laser systems - a main "slow" compression laser driver ( 20-40ns) and a separate fast, petawatt-class ignition laser ( $\sim 10$ ’s ps), whereas shock ignition would be accomplished with the standard NIF laser. Timing and spatial focusing requirements for shock ignition are also less demanding than those for fast ignition, while computer modeling involves only conventional radiation-hydrodynamics implosions on simple target configurations at standard laser intensities so that simulation results should be more tractable in terms of today's models and databases. Of course, shock ignition still requires ignition from a central, high temperature hotspot and thus conventional hydrodynamic symmetry and stability constraints will apply. Thus, as described below, major critical issues for shock ignition include convergence symmetry and stability of the shock pressure drive at the hotspot.

Parallel studies of shock ignition applied to the near term facilities HiPER and FTF have demonstrated the potential for high gain and yield for this new class of target $[12,13]$ and Ribeyre et al. have discussed the fielding of shock ignited targets at low drive energies on LaserMegajoule (LMJ) [12]. Like fast ignition, shock ignition offers the promise for high-gain ICF at low laser drive energies that may ultimately lead to smaller, more economic fusion power reactors and a cheaper fusion energy development path. Such advanced target concepts are now under consideration for future next-step inertial fusion energy facilities such as HiPER[12,14], FTF[13] and LIFE [15].

\section{Prospects for a Near Term High Gain Experiment}

We believe there is an opportunity to test a high gain shock ignited target on NIF at around $\sim 0.5 \mathrm{MJ}$ drive energy in the near-term ( $\sim 3-4$ years) with no significant new laser hardware required. This is due to its simple all-DT target design, its prospective in-flight 


\section{LLNL Technical Report LLNL-TR-428513, Revision 0 - April 23, 2010}

robustness, the potential need for only 1D SSD beam smoothing, little or no early time LPI preheat issues, and need for at most only minimal modifications to the NIF front end.

Table 1 outlines prospective routes to fielding shock ignition targets on NIF ranging from near term to longer term. The longer term options offer the possibility for very high gain targets that are fully fusion-reactor-relevant, but at the expense of longer lead times due to requirements for new laser hardware. In this paper, we concentrate on the potential for a near-term test on NIF (first column of Table 1). By near-term we mean an experiment that could be fielded with: (a) at most only minimal modifications to the NIF front end at the circuit board level, (b) no modifications to laser hardware or signal path routing from the pre-amplifiers to the final optics - that is, no hardware modifications in the path: PAMS $\rightarrow$ PABTS $\rightarrow$ main amplifiers $\rightarrow$ transport filters $\rightarrow$ conversion crystals $\rightarrow$ lenses $\rightarrow$ phase plates, and (c) no disruption to the baseline indirect drive ignition campaign.

Table 1. Options for high gain shock ignited target systems on NIF. The first column lists candidate parameters and requirements for a near-term ( 3-4 years) experimental test

\begin{tabular}{|c|c|c|c|}
\hline & $\begin{array}{c}\text { Near Term Target: } \\
\sim 3-4 \text { years }\end{array}$ & $\begin{array}{c}\text { Intermediate Term Target: } \\
\sim 4-6 \text { years }\end{array}$ & $\begin{array}{c}\text { Longer Term Target: } \\
\sim 5-10 \text { Years }\end{array}$ \\
\hline Target Type & All-DT & Wetted $\mathrm{CH}$ foam+DT & Wetted $\mathrm{CH}$ foam+DT \\
\hline $\begin{array}{l}\text { Drive Energy } \\
\text { (MJ) }\end{array}$ & $\sim 0.5(3 \omega)$ & $\sim 1-1.5(3 \omega)$ & $\geq 1.5(3 \omega),>2(2 \omega)$ \\
\hline Gain & $\sim 60$ & $\geq 100$ & $\geq 150$ \\
\hline Yield(MJ) & $\sim 30$ & $\geq 100$ & $\geq 200$ \\
\hline Drive geometry & Polar direct & Polar direct & Symmetric direct ${ }^{*}$ \\
\hline Phase plates & $\begin{array}{l}\text { Day-1 indirect drive } \\
\text { (partially defocused) }\end{array}$ & New direct drive CPP's & New direct drive CPP's \\
\hline $\begin{array}{l}\text { Drive uniformity } \\
\text { methods }\end{array}$ & $\begin{array}{l}\text { Repointing + defocus + } \\
\text { dynamic power } \\
\text { phasing/quad }\end{array}$ & $\begin{array}{l}\text { Repointing + dynamic power } \\
\text { phasing/quad }\end{array}$ & $\begin{array}{l}\text { Symmetric direct }{ }^{*}+\text { power } \\
\text { phasing/quad }\end{array}$ \\
\hline $\begin{array}{l}\text { NIF focusing } \\
\text { architecture }\end{array}$ & $\begin{array}{l}2 \text { out of } 4 \text { quads/beams } \\
\text { focused at rShock }\end{array}$ & $\begin{array}{l}1 \text { out of } 4 \text { beams focused at } \\
\text { rShock }\end{array}$ & $\begin{array}{l}1 \text { out of } 4 \text { beams focused at } \\
\text { rShock }\end{array}$ \\
\hline $\begin{array}{l}\text { Peak shock } \\
\text { power (TW) }\end{array}$ & $\sim 300-350$ & $\geq 350$ & $\geq 400(3 \omega), \sim 600(2 \omega)$ \\
\hline Beam smoothing & $\begin{array}{l}\text { Multi-FM 1D SSD } \\
\text { Day-1 tripler crystals }\end{array}$ & $\begin{array}{l}\text { Multi-FM 1D SSD } \\
\text { Day-1 tripler crystals }\end{array}$ & $\begin{array}{l}2 \mathrm{D} \mathrm{SSD} \\
\text { Dual tripler crystals }\end{array}$ \\
\hline Bandwidth(THz) & $0.25-0.5$ & 0.5 & $1.0^{*}$ \\
\hline
\end{tabular}

* Not needed if high gains can be achieved with PD+multi-FM SSD @ $\leq 0.5 \mathrm{THz}$ 


\section{LLNL Technical Report LLNL-TR-428513, Revision O - April 23, 2010}

Compared to conventional high-aspect-ratio, high velocity, hotpot ignition targets, shock ignited targets are characterized by stable, low-aspect-ratio thick shells that should be less susceptible to both initial speckle imprint (may need only 1D SSD) and hot electron preheat. Thus, these platforms may be easier targets to field and do not necessarily have to be preceded by demonstration of conventional (polar) direct drive ignition. The exploration of shock ignition on NIF could then proceed in parallel with both the indirect drive National Ignition Campaign and conventional polar direct drive .

\section{Target Design, Fabrication and Fielding}

An initial candidate NIF shock ignition target is shown to scale in Fig. 3, together with a preliminary set of 1D performance parameters. This target build is appropriate for 1D gains $\sim 60$ at $\sim 0.5 \mathrm{MJ}$ total drive energy drive (i.e., $\sim 30 \mathrm{MJ}$ yield). Considerable $2 \mathrm{D}$ and $3 \mathrm{D}$ studies are now required to validate low mode symmetry and higher mode stability.
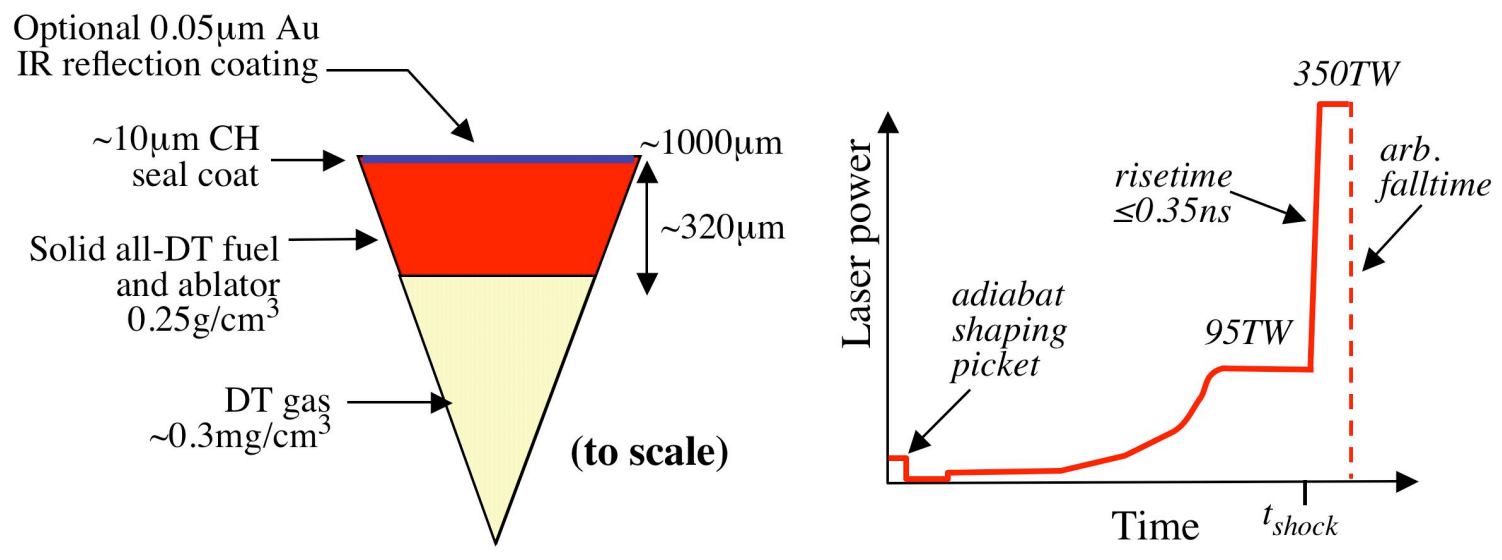

\begin{tabular}{|ll|}
\hline - Yield , gain & $33.4 \mathrm{MJ}, 60.4$ \\
- Drive energy: main + shock & $0.253+0.5=0.553 \mathrm{MJ}$ \\
- Peak powers: main, shock & $95 \mathrm{TW}, 350 \mathrm{TW}$ \\
- Ignition window at Yield & $50 \%$ \\
- Marginal shock energy, power & $400 \mathrm{ps}$ \\
- IFAR(66\%) & $0.14 \mathrm{MJ}, 260 \mathrm{TW}$ (for $\mathrm{Y} \geq 30 \mathrm{MJ})$ \\
- $\mathrm{V}_{\max }:$ main only, main+shock & 15.3 \\
- CR: $\mathrm{r}_{0} / \mathrm{r}_{\mathrm{HS}}$ (conv defn), $\mathrm{r}_{\text {gas }} / \mathrm{r}_{\mathrm{HS}}$ & $2.54 \mathrm{e} 7,2.98 \mathrm{e} 7 \mathrm{~cm} / \mathrm{s}$ \\
\hline
\end{tabular}

Fig. 3. Spherical radial build (to scale), schematic pulse shape and preliminary $1 D$ performance parameters for one candidate all-DT NIF shock ignition target for nominal gain $\sim 60$ (30MJ yield) at a total drive energy of $\sim 0.5 \mathrm{MJ}$. These parameters were obtained by in $1 D$ but employing $3 D$ laser ray trace to obtain beam deposition efficiencies. 
The target consists of a simple, thick shell of frozen DT that forms both fuel and ablator surrounded by a thin $(\sim 10 \mu \mathrm{m}) \mathrm{CH}$ seal coat. The low initial aspect ratio of $\sim 2.7$, corresponding thick shell $(\sim 320 \mu \mathrm{m})$ and low implosion velocities can result in high fusion gains because, compared to the higher aspect ratio thin shells of conventional, high velocity direct or indirect drive targets, considerably more fuel mass can be assembled for a given laser drive energy. Consequently, this platform is characterized by beneficially low peak velocities, low Atwood numbers (no fuel/ablator density mismatch) and low inflight aspect ratios (IFARs) around 15 (Fig. 3). Such targets should then exhibit good hydrodynamic stability during the acceleration phase such that Rayleigh-Taylor (RT) growth of outer surface perturbations is unlikely to penetrate the shell during implosion.

Thin-walled plastic $\mathrm{CH}$ shells required for the all-DT target in Fig 3. have been fabricated in the laboratory [16] with specifications that are as good or better than those for the thick-walled NIF indirect drive ignition target, while LLE/University of Rochester have already made all-DT cryogenic targets with thickness comparable to those required here (see Fig. 4.)

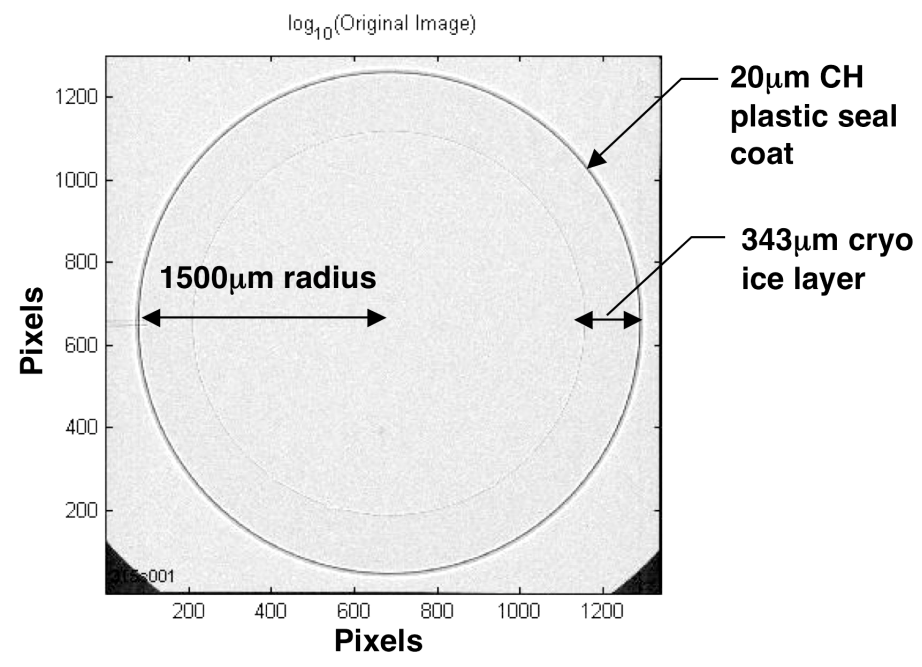

Fig 4. X-ray phase contrast image of an all-DT cryo target with outer CH seal coat (courtesy D. Harding LLE/U.Rochester)

An alternative target design of DT fuel with an outer ablator comprising DT wicked into low density $\left(\sim 100 \mathrm{mg} / \mathrm{cm}^{3}\right) \mathrm{CH}$ foam could be considered in the longer term (see Table 1). Such a target would likely exhibit higher fusion gain due to higher laser coupling efficiency (see blue points in Fig. 2) but at the expense of more complex target fabrication and issues of shell foam roughness with attendant stability concerns. Such 
wetted foam ablators should thus be considered only as future options or to ameliorate possible TPD problems (see below).

LLE/University of Rochester are investigating cryostat configurations for fielding direct drive cryo targets on NIF. Their objective is a plug-and-play design that mates directly with the cold plate at the end of the present NIF target positioner. Like the baseline indirect drive ignition target, DT filling of the shock ignition target in Fig. 3 would be accomplished by a fill tube (no diffusion-fill capability on NIF in the near term); but unlike those targets this all-DT design may incur little or no 3D stability penalty because of the relatively thin seal coat with no extension of the fill tube into the DT ablator. With (polar) direct drive (see below), the target would be supported on the fill tube stalk inside a simple cryostat cooled by internal He gas flow. However, although we avoid the complexity of a hohlraum cryostat, we then lack the latter's thermal mass and IR reflection properties which means that, to avoid a deleterious temperature rise, the target would have less residence time between the opening of the cryo shroud and the start of the laser pulse. Typically, the thinner the outer $\mathrm{CH}$ seal coat and the thicker the DT ablator/fuel ice layer, the longer the permitted residence time. There is also the option to coat the outside of the $\mathrm{CH}$ shell with a few-hundred-Angstrom IR reflection layer of gold (Fig. 3). This feature may also ameliorate early time laser imprint as ablation of the high-z layer with the initial picket produces $\mathrm{x}$-rays that expand the $\mathrm{CH}$ seal layer to enhance standoff smoothing; experimental observations of this process were reported in Ref. 17. The current NIF target positioner arm takes $\sim 3$ s to ring down after the opening of its co-located clamshell cryo shroud. We are presently studying whether the thick all-DT target of Fig. 3 with the outer Au IR reflection layer can meet this time specification. If not, we may need to employ an opposed-port cryo shroud; this was originally designed for the indirect drive ignition target but has not been implemented to date.

\section{NIF Beam Geometry - Optimization of Polar Drive Symmetry}

Near term shock-ignited targets would be fielded on NIF under the polar-directdrive campaigns envisaged for standard NIF direct drive targets $[5,6,7,18,19]$ but would employ day-1 indirect drive phase plates [20]. A key immediate need, therefore, is to 


\section{LLNL Technical Report LLNL-TR-428513, Revision 0 - April 23, 2010}

verify the adequacy of low mode drive uniformity and shock symmetry under polar drive for the convergence ratios predicted for these shock ignited targets.

Our present simulations indicate that it may not be possible to achieve shockignition on NIF in a conventional indirect drive hohlraum because, while the laser can supply the required fast rise of the shock pulse (see below), there is a time lag in conversion of laser energy to radiation temperature due to the hohlraum heat capacity. Thus the radiation drive rises too slowly to achieve required shock synching relative to the hydro bounce of the stagnating fuel. In addition, direct drive is typically a factor of 2.5-3-times more efficient than indirect drive in terms of the fraction of laser drive energy imparted to the converging shell at peak kinetic energy.

Under polar-drive (PD), the NIF beams are retained in the present indirect drive port configurations but adequate direct drive uniformity is potentially achievable by a combination of repointing, partial defocusing of some quads and phasing the relative time-dependent power balance from quad to quad. Wetted $\mathrm{CH}$ foam ablator targets under NIF polar drive at $1 \mathrm{MJ}$ are predicted to achieve significant $2 \mathrm{D}$ gain in the presence of all NIF-specific sources of perturbation [6,7]. PD tests performed on Omega in NIFgeometry-relevant experiments have shown that polar drive is able to compensate for the low-mode uniformities [18]. More recent Omega experiments with exploding-pusher gasfilled $\mathrm{SiO}_{2}$ targets gave $\sim 89 \%$ of the predicted clean 1D yields with the beams in full $4 \pi$ symmetric configuration and retained $80 \%$ of this performance when switched to the polar drive configuration [21]. PD tests are scheduled for NIF later in 2010 on gas-filled $\mathrm{SiO}_{2}$ capsules designed to produced nuclear yield for diagnostic calibration but, like the above Omega experiments, these targets will have low convergence ratios.

Given the shock pulse is launched at late time where the capsule has converged around a factor of $\sim$ three from its original diameter, we can consider optimizing the shock-drive absorbed power by devoting a subset of NIF quads/beams to the shock pulse such that those selected beams are focused at the converged capsule radius at that time. Fig. 5 shows an initial option for this where half the quads ( 96 beams) are focused at the initial capsule radius at time zero while the other half (96 beams) are focused at the reduced capsule radius at time of shock launch at late time in the drive. Such "zooming" may enhance the shock-drive intensity and provide enhanced coupling of the shock 
pressure pulse to the compressed core. In addition each quad can be split in up to four independently pointed/focused beams, thus resulting in a total of 8/16 rings of quads/beams, top and bottom.
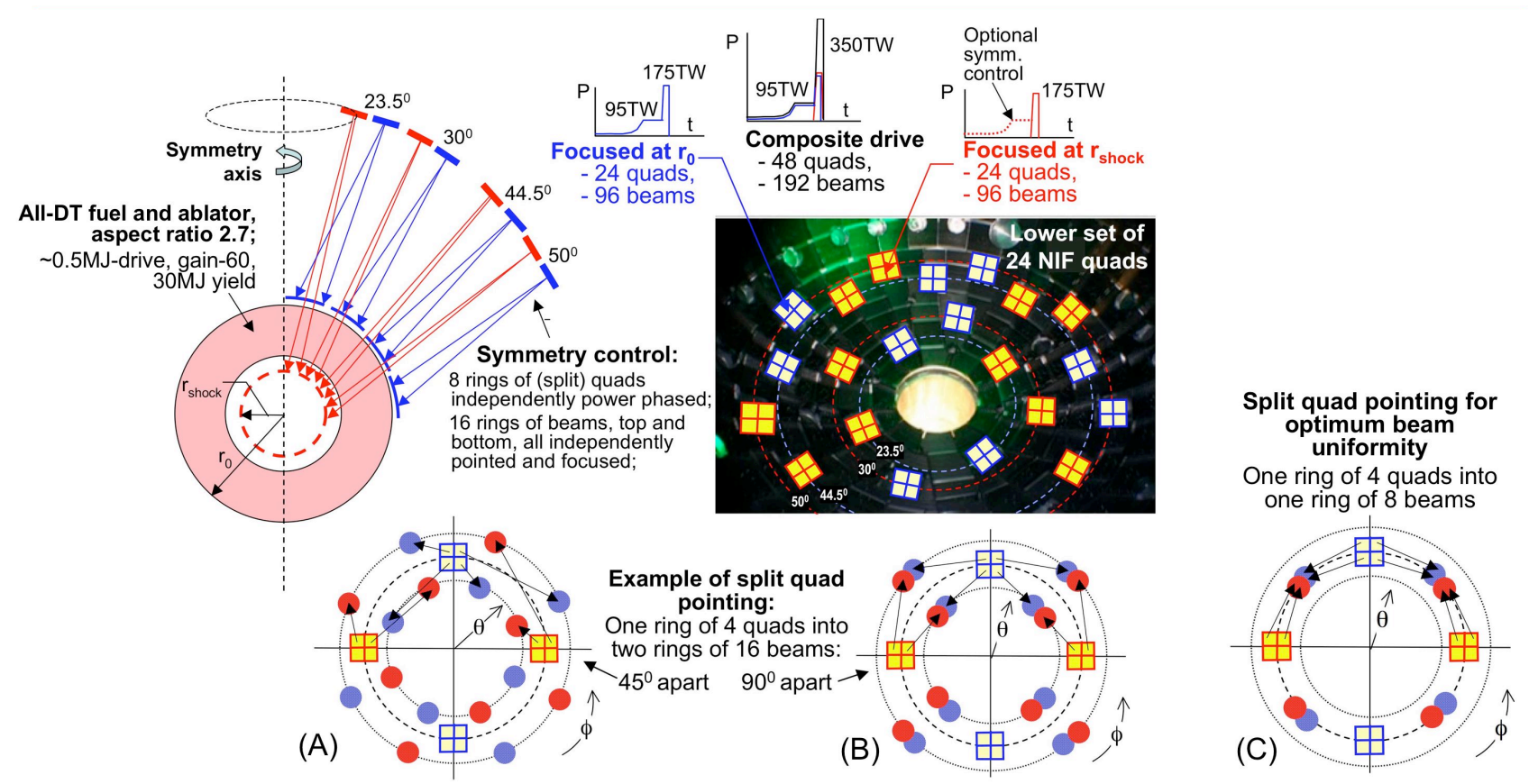

Fig 5. Initial "zooming" options for optimizing polar drive symmetry for the candidate $0.5 M J$ all-DT shock ignited target. Half the quads (96 beams) are focused at the capsule radius at time zero while the other 96 beams are focused around the reduced critical radius at time of shock launch. Split quad pointing and focusing can be employed to optimize beam uniformity in both polar and azimuthal angles. Options $A$ and B provide a total of 8 rings of (split) quads and 16 rings of beams, top and bottom. Option $C$ provides only 4 rings of (split) quads and 8 rings of beams, top and bottom, but with better compensation for any beam-to-beam nonuniformity within a quad

An alternative polar drive configuration is shown in Fig. 6. Here, we give up late time zooming of the shock pulse, such that all beams are focused around the initial capsule radius at $\mathrm{t}=0$. This configuration also has the option for either partial split-quad focusing/pointing or none; in the latter case, all beams from one quad are pointed at the same focal spot. This geometry may be a preferred option if the present NIF beam-tobeam power balance uniformity is not sufficient to achieve symmetry requirements. However, this beam balance issue could be separately resolvable by exchanging conversion crystals from quad to quad so that each beam in a given quad has the same optics chain; this would require some machine downtime $\sim 1-2$ weeks for the retrofit. 


\section{LLNL Technical Report LLNL-TR-428513, Revision O - April 23, 2010}

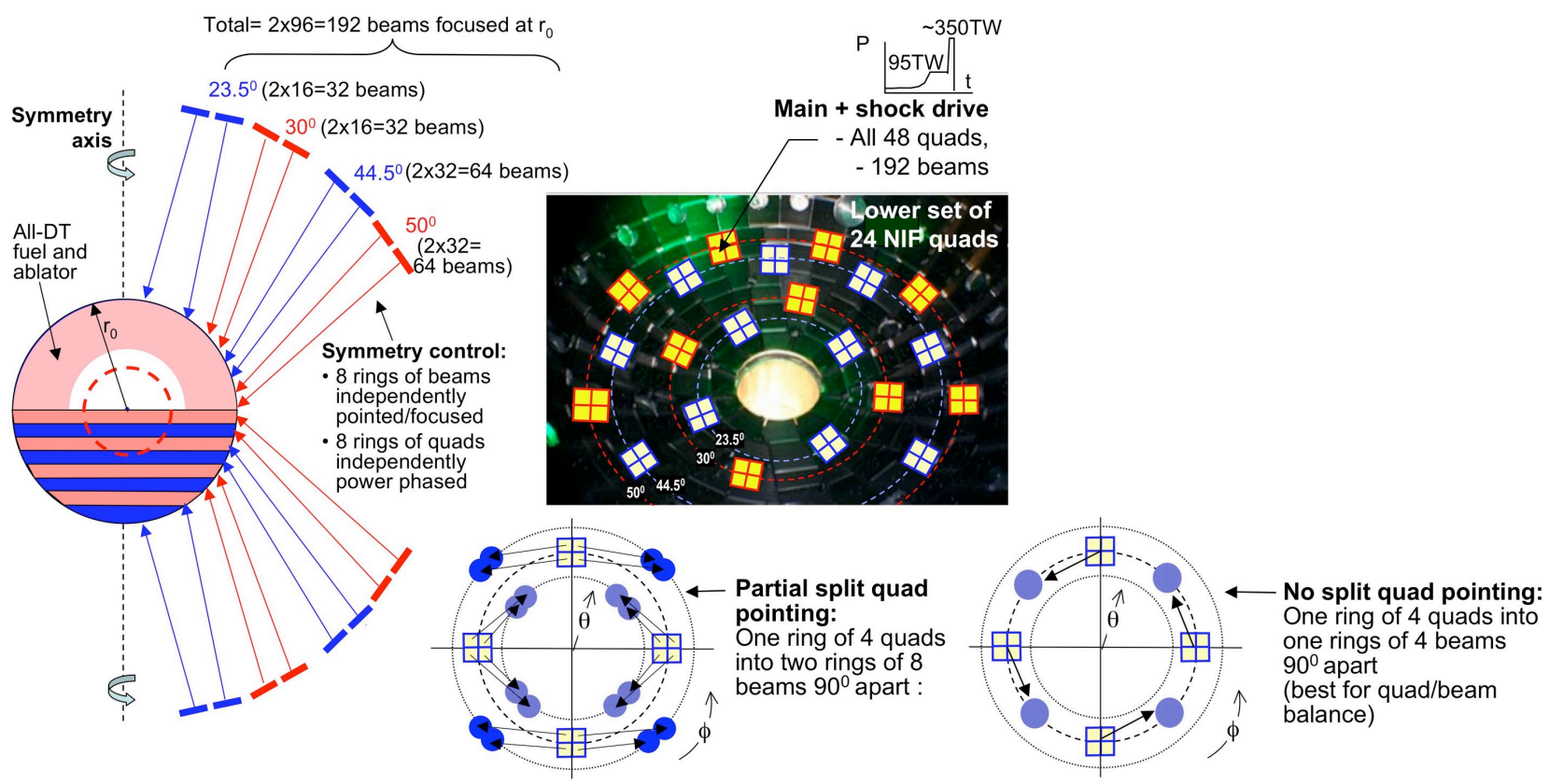

Fig 6. Alternative configuration for polar drive symmetry for shock ignition for optimum beam-to-beam power balance within a given quad. All beams/quads are focused at the capsule radius at time zero, with the options of partial or no split quad pointing/focusing. The result is a total of 8/4 rings of partial-split/unsplit quads and 8/16 rings of beams, top and bottom.

Design studies in 2D are now in progress to determine an optimum laser drive specification for configurations such as those in Figs. 5 and 6 - that is, optimization of beam pointings, focusing and power phasing per quad - to achieve adequate low mode symmetry. Formally, these studies comprise an outer iteration to determine an optimum set of beam pointings and focusing and an inner iteration to determine the relative power phasing control of each azimuthal ring of quads as a function of polar angle. Note that because our present gain-60 baseline target has modest drive energy $(\sim 0.5 \mathrm{MJ}$ in $1 \mathrm{D}$ at a maximum power of $\sim 350 \mathrm{TW}$ ) we have energy and power headroom to spare when tuning polar-dependent power relative to the nominal requirements. These margins are discussed further in Section 7 below

One candidate approach from Murakami [22] assesses beam pointing/focusing irradiation uniformity via a power spectrum as a function of Legendre modes. The critical parameter is the lowest dominant mode $n_{d}$; lower mode uniformities below this can be suppressed if such imperfections such as beam-to-beam (or quad-to-quad) power imbalance and pointing error can be suppressed at a sufficiently low level. Other uniformities for higher mode numbers $n>n_{d}$ then decay exponentially. The strategy is to 


\section{LLNL Technical Report LLNL-TR-428513, Revision 0 - April 23, 2010}

maximize $\mathrm{n}_{\mathrm{d}}$ under a fixed number of beams $N_{B}$ where generally $n_{d}$ increases with the total number of beams; for example, $n_{\mathrm{d}}=4,6$, and 10 , for $N_{B}=4$ (tetrahedron), 12 (dodecahedron), and 32 (20 vertices and 12 face centers of dodecahedron), respectively. The procedure would be determined by the specific geometry for optimization, i.e., cylindrical [23] or spherical [24] although the latter (spherically symmetric) geometry would not be available on NIF in the near term (See Table 1). .

Depending on the number of independent variables, the outer iteration to determine an optimum set of pointings/focusings can involve a full or partial grid search, heuristic optimization techniques such as genetic algorithms, or simply sensitivity studies around a set of mean values; they can be performed under direction of an optimization control shell such as DAKOTA [25]. Selection of one given pointing/focusing geometry is followed by a full 2D (ultimately 3D) hydro implosion simulation to assess later time capsule symmetry. Note that, in principle, the inner iteration to determine relative power phasing per quad - in $2 \mathrm{D}$, this reduces to the relative power phasing per polar quad ring only requires one implosion simulation. Specifically, it may be possible to tune "on-thefly" at each time step of the hydro code by adjusting each quad laser power profile relative to the reference profile from the $1 \mathrm{D}$ simulation in order to radially synchronize a datum surface - e.g., the critical surface, peak density gradient surface, or surface of peak laser deposition, etc. For example, fitting to the datum surface with a low mode spherical harmonic and using the laser ray depositions in the zones where this surfaces lies, we can compute each laser's contribution to the modes of the expansion. With more lasers than modes, this can be treated as a least-squares problem in the laser powers needed to damp the modes. Thus at the end of the simulation, the required time-dependent laser power profile in each quad ring has been derived. Whether such a procedure is sufficiently stable in one forward implosion run remains to be determined.

Once a candidate polar drive geometry is established from 2D studies, an immediate term issue is to perform proof-of-principle tests on NIF with surrogate, hydroequivalent room-temperature $\mathrm{CH}$ shells containing diagnostic gas fill. 


\section{Beam Smoothing and Higher Mode Stability}

Higher mode beam imprint at early time is an important issue for direct drive as, together with outer surface roughness, it forms a seed for later-time Rayleigh-Taylor (RT) growth [26]. Shock ignited NIF targets may be less susceptible to imprint relative to regular direct drive hotspot ignition targets because of the thicker low aspect ratio shell which is resistant to outer surface perturbations and in-flight breakup (IFARs are only $\sim 15$ ) and, possibly, the potential for enhanced ablative stabilization from later time SRSgenerated hot electrons (see below).

Ideally, 2D SSD (smoothing by spectral dispersion) at a bandwidth of $1 \mathrm{THz}$ would be implemented to smooth the laser speckle for conventional higher aspect-ratio direct drive targets [4] but would necessitate modifications to all 48 NIF preamplifier modules (PAMs) plus the employment of dual tripler crystals at the final focus. Thus, this is probably not an option for a near-term experimental test of shock ignition.
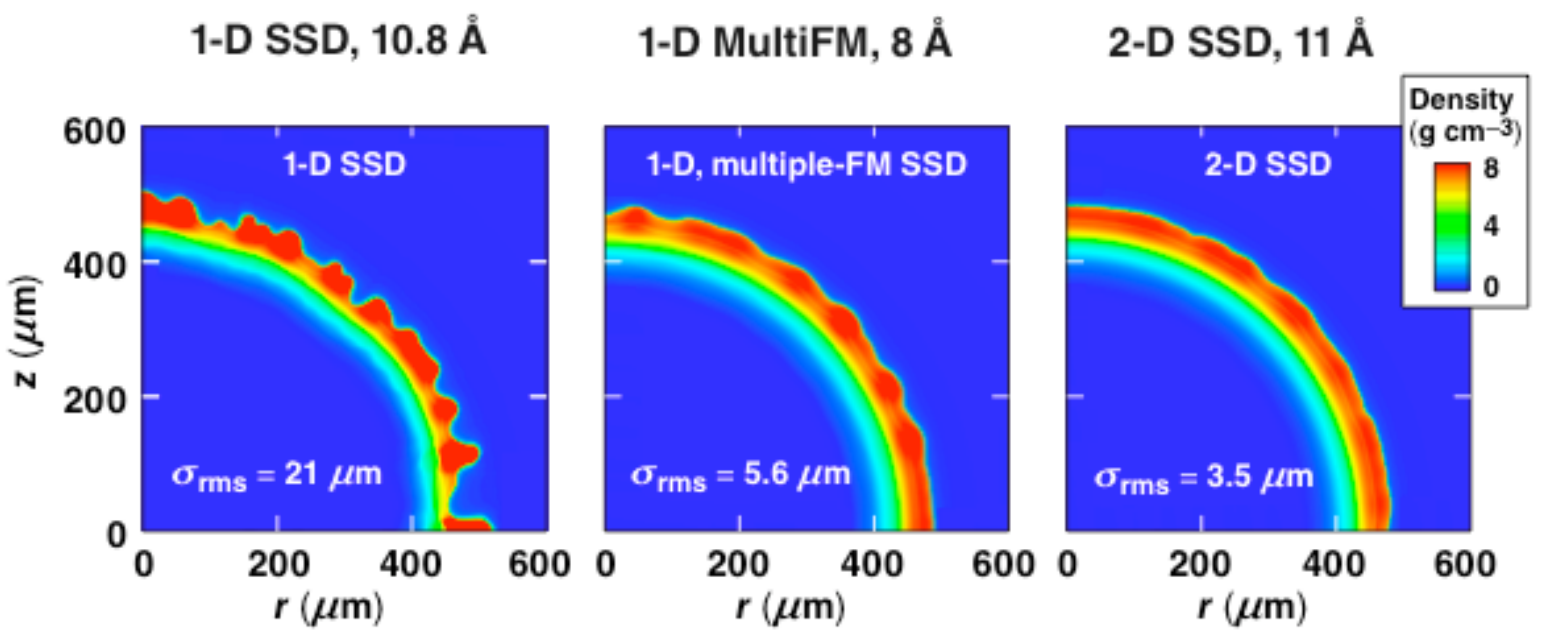

Fig. 7. Simulations of multi-FM ID SSD beam smoothing on a candidate NIF $1.5 M J$ drive $C H$ foam/DT target. Late time imprint at the end of the acceleration phase reduces to $2 D$ SSD levels (from Ref. 28)

An alternative near-term beam smoothing scheme for NIF is currently under investigation at LLE and employs simple 1D multi-FM SSD at $\leq 0.5 \mathrm{THz}[27,28]$. Compared with 2D SSD, it has reduced complexity (no second dimension), is applied in a fiber in the front end rack mount unit (no new bulk optics required in the PAMs), takes advantage of multiple color cycles without the disadvantage of coherence maxima in the spectrum, and would use the present day-1 tripler crystals. As shown in Fig 7, 


\section{LLNL Technical Report LLNL-TR-428513, Revision O - April 23, 2010}

simulations by Marozas et al [28] indicate that imprint reductions are comparable to 2D SSD in direct drive and will be applicable to polar direct drive. Experimental validation in 2010-2011 at LLE/University of Rochester will be an important test of this method.

Preliminary 2D higher mode stability studies have also been performed on shock ignition implosions by Betti et al [29] and Ribeyre et al. [12]; they suggest that the stability of the hotspot boundary can be quite insensitive to RT at stagnation due to nonlinear interference between RT and a stabilizing opposite-phase Richtmyer-Meshkov stage. In general, multimode calculations of high mode beam imprint and inner ice roughness are an important data need in parallel with the lower mode symmetry studies

\section{Laser Plasma Interactions}

Because of high laser intensities during shock launch $\left(\sim\right.$ several $\times 10^{15} \mathrm{~W} / \mathrm{cm}^{2}$ to greater than $10^{16} \mathrm{~W} / \mathrm{cm}^{2}[3]$ ), a potential concern for NIF shock ignition is the onset of laser-plasma instabilities (LPI) including stimulated Brillouin scattering (SBS), stimulated Raman scattering (SRS) and two-plasmon decay (TPD). SRS and TPD can result in the generation of suprathermal electrons which, for conventional NIF direct and indirect drive hotspot targets, can be a serious source of preheat in the precompressed fuel as soon as the laser approaches its main drive power.

By contrast, it is important to note that in shock ignition the high laser intensity is not applied until late time where the fuel is approaching stagnation. Thus, the now dense imploding shell is capable of absorbing SRS or TPD-generated hot electrons up to high energies, shielding the inner DT fuel from preheat [1, 3, 12, 29,30]. Moreover, providing their energies are less than $\sim 100 \mathrm{keV}$, generation of such hot electrons might actually enhance shock drive performance due to increased ablation pressures, strong ablative stabilization of R-T instabilities and symmeterization of the converging shock pressure front [3]. Of course, with its relatively low onset threshold, TPD must also be monitored at earlier times during the low intensity main pulse. If this should prove to be a substantive issue then recourse may then be necessary to the use of a wetted-foam $\mathrm{CH}$ ablator or all-CH plastic ablator to shield the main DT fuel.

New Fokker-Planck calculations exploring non-local hot electron transport relevant to shock ignition [31] indicate that the relatively collisionless energetic electrons 


\section{LLNL Technical Report LLNL-TR-428513, Revision O - April 23, 2010}

have long path lengths around the surface of the target and that the tangential heat flow around the target is comparable with the radial heat flow into the target even though the temperature gradients are more gentle. In particular, even with two-sided asymmetric laser drive, the drive pressure appeared quite uniform.

Recent shock-ignition-relevant experiments on Omega at NIF-relevant intensities on low adiabat $\mathrm{CH}$ shells containing $\mathrm{D}_{2}$ gas showed a factor of $20 \mathrm{X}$ increase in neutron yield on application of the shock pulse [30]. While not a test of shock ignition per se, the experiments demonstrated that compression assembly and shock pulses can be successfully synchronized and the results illustrated a significant improvement in the performance of low-adiabat, low velocity implosions compared to conventional hotspot implosions. In these experiments, approximately 35\% LPI backscatter was seen at a shock laser intensity of $\sim 1 \times 10^{16} \mathrm{~W} / \mathrm{cm}^{2}$, reducing to about $20 \%$ at $\sim 5 \times 10^{15} \mathrm{~W} / \mathrm{cm}^{2}$. At the highest intensities, the reflection was dominated by SRS with a smaller contribution from SBS; there was no detectable contribution from TPD. A SRS hot electron population of $\sim 10 \%$ of the incident energy was generated around $40-45 \mathrm{keV}$, with temperature independent of laser intensity. As above, we believe hot electrons in this energy range may be beneficial to both the hydro efficiency and symmetry of shock drive for NIF targets. Note also that our candidate near-term NIF design (Fig. 3) has total drive energies of only $\sim 0.5 \mathrm{MJ}$ and is not power limited in the shock spike (see below), so reductions in absorbed energy due to increased backscatter can be compensated by increases in shock launch power.

A European collaboration KITSI-"KInetics for Shock Ignition"- has commenced on electron transport modeling in the shock ignition context [32]. The goal is to run full Vlasov Fokker Planck and PIC simulations of energy transport during the spike. This work is oriented to HiPER [14] for both fast and shock ignition applications but we envisage strong collaboration with this initiative for NIF shock ignition applications.

\section{NIF Energy, Power and Timing Requirements}

Fig. 8 provides an idea of the nominal peak power and energy capability of NIF at $3 \omega$; actual maximum performance limits will be determined by the precise pulse shape required. Shock ignited targets on NIF have the potential to realize high gain at modest 
$\sim 0.5 \mathrm{MJ}$ drive energies ( $\leq 350 \mathrm{TW}$ peak powers) - i.e., around one-third the total energy capability of NIF at $3 \omega$ - and would enable us to take advantage of the lower ensuing damage thresholds in NIF optics with greater shot budgets.

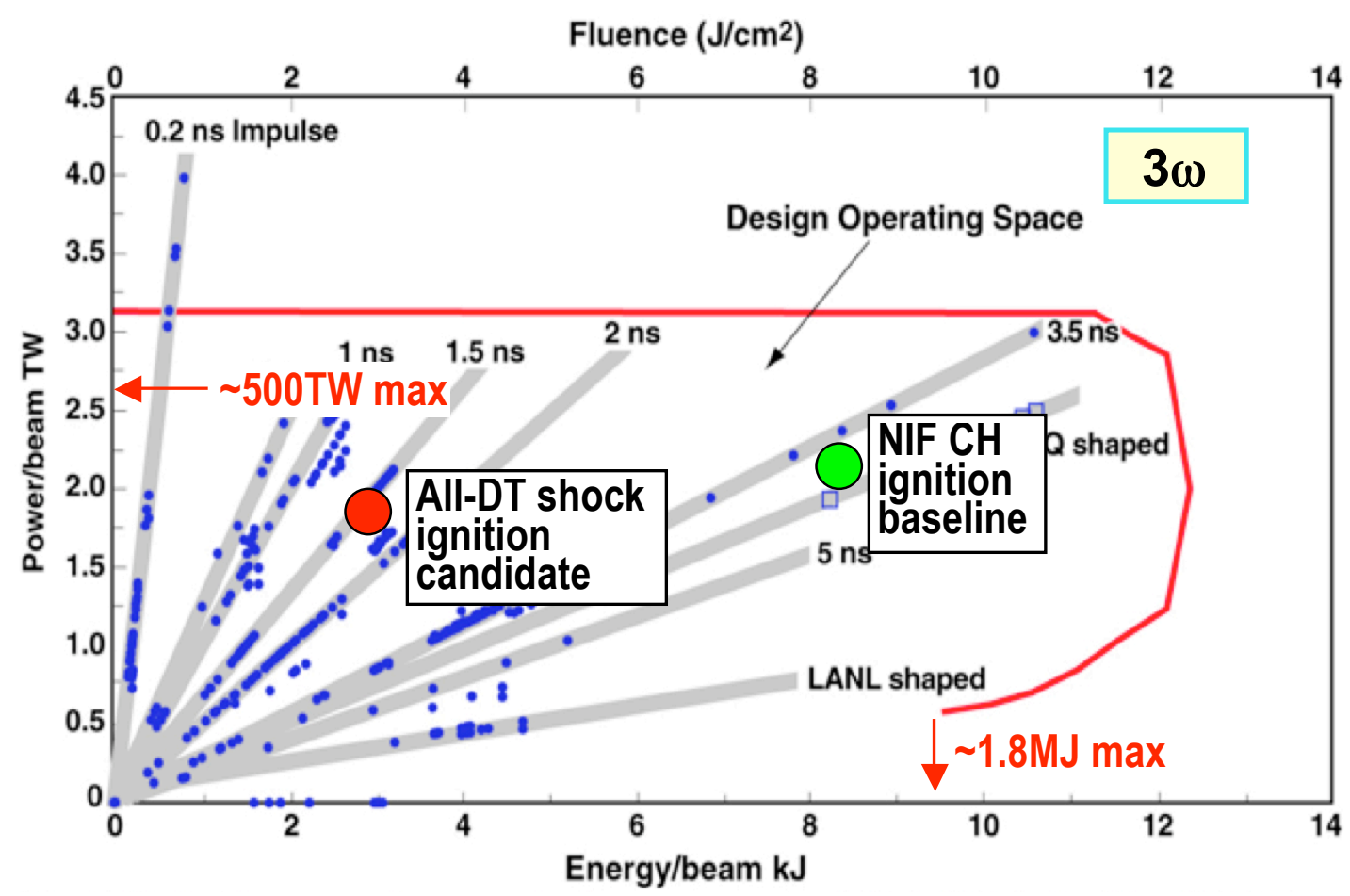

Fig. 8. Nominal power and energy operating space for NIF at $3 \omega$. Actual operating limits will depend on the pulse shape required. A near term shock ignition candidate target will require $\sim 0.5 \mathrm{MJ}$ at peak powers of $\sim 350 T W$ ( red point)

In terms of laser power requirements, whereas the main drive pulse for fuel assembly will be at a modest peak power $P_{\text {main }} \sim 95 \mathrm{TW}$, the shock pulse $P_{\text {shock }}$ will require higher peak powers of $\sim 300-350 \mathrm{TW}$. For comparison, the baseline NIF indirect drive ignition target with $\mathrm{CH}$ ablator requires equivalent peak powers/beam of $\sim 415 \mathrm{TW}$ at $\sim 1.6$ MJ drive energy. These are relative to maximum NIF peak powers at $3 \omega$ of $\sim 450-500 \mathrm{TW}$ (Fig. 6) depending on scenario. We have performed initial validations of our preliminary shock ignition pulse shapes with the NIF Laser Performance Operations Model [33]; results indicate that temporal contrasts should be achievable in the main amplifiers and that the proposed pulse shapes do not pose any equipment protection issues.

The shock launch time $t_{\text {shock }}$ (see Fig.1 above) determines the arrival of the shock ignition pulse relative to the hydro bounce of the stagnating fuel. For the candidate target in Fig. 3, ignition shock synching requires a shock pulse launch window - that is, the permissible spread of $t_{\text {shock }}-$ of $\sim 0.4 \mathrm{~ns}$ with a rise-time requirement of $\leq 0.35 \mathrm{~ns}$. It is not 
clear at this time whether such specifications will necessitate any modifications to the NIF front-end arbitrary waveform generators (AWG).

We have performed an initial attempt at generating the required fast rise as shown in Fig 9. Here a second slicer in the AWG is employed to give a fast internal rise time; the dip in the pulse just before the fast rise is caused by the first slicer turning off and the second slicer turning on. The $10 \%-90 \%$ rise time is $\sim 100 \mathrm{ps}$, a factor of three better than our requirement. The shock pulse is to be maintained at peak power for $\geq 0.8 \mathrm{~ns}$ to deliver the required shock drive energy $(\leq 0.3 \mathrm{MJ})$ but can held on for any arbitrary time after that, i.e., there is no requirement for a fast fall time as the target would have already reached ignition conditions. Any structure before the rise or at the flat top is probably of no consequence as the shock pulse is merely required to deliver an intense pulse of energy in a given time window.

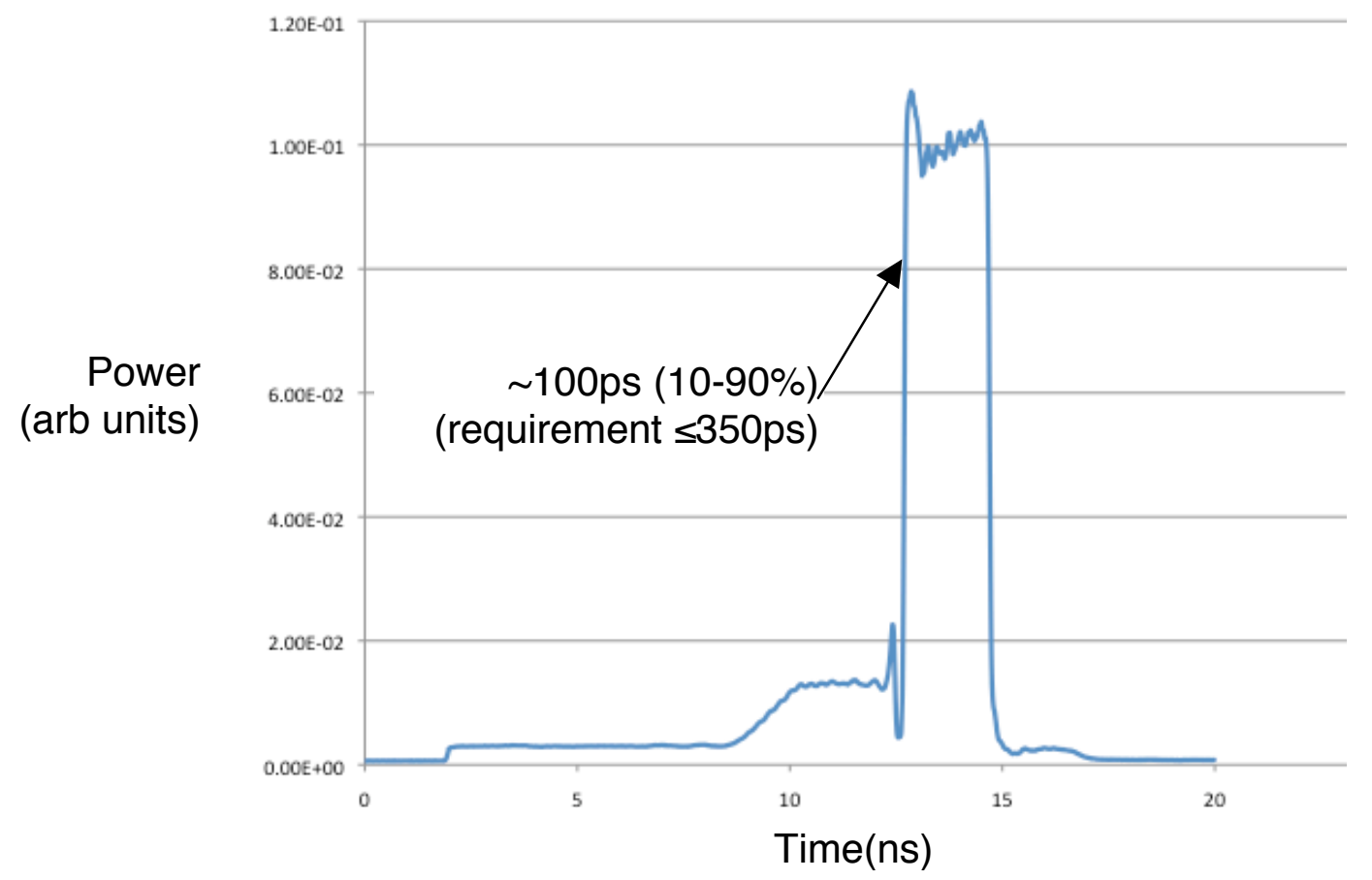

Fig. 9. Initial attempt at forming the risetime of a NIF shock ignition pulse shape in the NIF AWG. The 10\%-90\% power rise time is around 100ps, a factor of three better than the $\leq 0.35$ ns required. There is no requirement for a fast fall time at the end flattop as the target would already have reached ignition conditions

Accordingly, we believe the hardware may be already capable of generating internal rise times that are acceptable for shock ignition; controller software may need 
modifications. But should any hardware upgrades to the AWGs be required these will likely be only low cost items at the circuit board level.

\section{Integrated Work Plan for Experimental Planning and Fielding}

In conclusion, we believe there is an opportunity to test the feasibility of a high gain, shock ignition target on NIF in the near term without the need for new laser hardware. In the work plan of Fig. 10 we show the prospective tasks that must be performed in order for such targets to be fielded. The items shown in solid red denote critical path modeling and analysis tasks that should be tackled in the immediate term for this concept to proceed to a formal experimental plan.

- Critical path anal., modeling \& design -General anal. \& design. $\quad$-Hardware and fabrication.

-Experiments

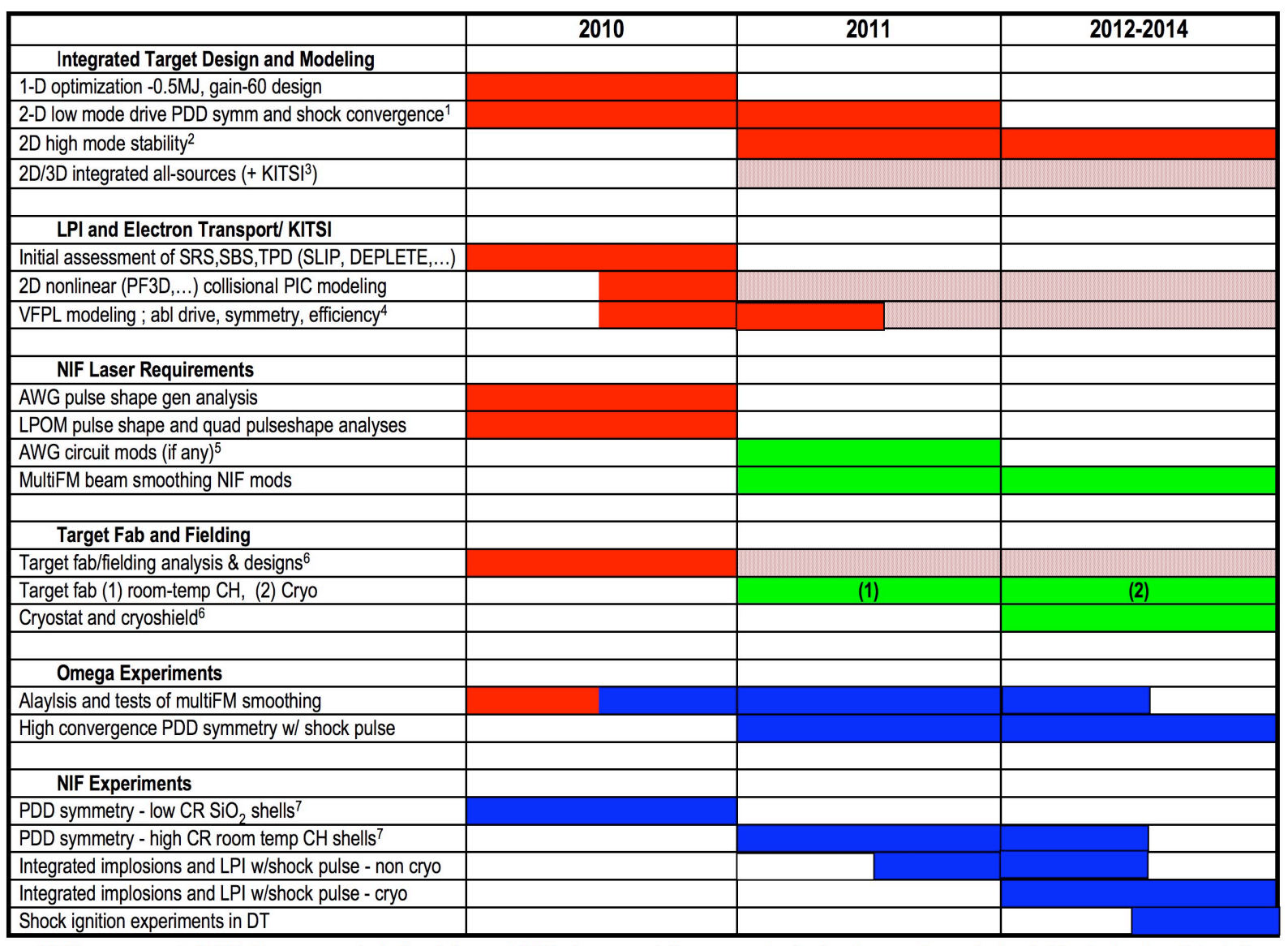

1-With assessment of PDD drive sources, including defocused CPPs, beam repointing, power phasing/quad, separate quads devoted to shock pulse. 2.-Including multiFM beam smoothing. 3-including LPI hot electrons - integration of KITSI PIC and VFPL models into hydrocodes. 4-Potential enhanced ablation pressure, ablative stabilization, smoothing and symmeterization from SRS hot electron . 5 - Only if necessary and at cct board level. 6-Incuding assessment of need for opposed port cryo shroud and construction thereof. 7-Using day-1 indirect drive phaseplates

Fig. 10. Candidate work plan leading to a feasibility test of a high gain, shock ignition target on NIF in the near term. The items shown in solid red denote critical path modeling and analysis tasks that should be tackled in the immediate term for this concept to proceed to a formal experimental plan. 


\section{Acknowledgements}

We are pleased to acknowledge informative discussions with: J.Nuckolls, J.Heebner, C.Haynam,, J.Zuegel, J.Marozas, S.Dixit, E.Mapoles, C.Edwards, B.Kozioziemski

\section{References}

[1] R. Betti , C.D. Zhou , K.S. Anderson , L.J Perkins, A.A. Solodov, "Shock Ignition of Thermonuclear Fuel with High Areal Density”, Phys. Rev. Lett., 98, 155001 (2007)

[2] J.D. Lindl, Inertial Confinement Fusion: The Quest for Ignition and Energy Gain Using Indirect Drive (New York: AIP Press, Springer 1998)

[3] L.J. Perkins, R.Betti, K.N. LaFortune, W.R., Williams, "Shock Ignition: A New Approach to High Gain Inertial Confinement Fusion on the National Ignition Facility", Phys. Rev. Lett., 103, 045004 (2009)

[4] T. J. B. Collins, J. A. Marozas, R. Betti, D. R. Harding, et al, "One-Megajoule, wetted-foam target-design performance for the National Ignition Facility", Phys. Plasmas 14, 056308 (2007)

[5] S. Skupsky, J. A. Marozas, R. S. Craxton, R. Betti, et al., "Polar direct drive on the National Ignition Facility”, Phys. Plasmas 11, 2763 (2004)

[6] J. A. Marozas, F. J. Marshall, R. S. Craxton, I. V. Igumenshchev, , et al., "Polardirect-drive Simulations and Experiments" Phys. Plasmas 13, 056311 (2006)

[7] P.W. McKenty, et al., "Mulitidimensional Numerical Investigation of NIF Polar Drive Designs with Full Beam Smoothing”, Inertial Fusion Sciences and Applications (IFSA) Conference, Kobe, Japan (2007)

[8] A. Maschke, IEEE Trans. Nucl. Sci., NS-22 No.3 1825 (1975)

[9] N. G. Basov, et al., J. Sov. Laser Research 13, 396 (1992)

[10] M. Tabak, J.Hammer, W.Glinsky, W.Kruer, S.Wilks, "Ignition and High Gain with Ultra Powerful Lasers”, Phys. Plasmas, 11626 (1994)

[11] Fusion Sci \& Technol 49 (2006) - Special issue on Fast Ignition

[12] X. Ribeyre, G. Shurtz, M. Lafon, S. Galera, S. Weber, "Shock Ignition: an Alternative Scheme for HiPER”, Plasma Phys. Control. Fusion, 51015103 (2009)

[13] A. J. Schmitt, J.W. Bates, S.P. Obenschain, S.T. Zalesak, D.E. Fyfe, R. Betti, "FTF Direct Drive Fusion Energy Shock Ignition Designs for Sub-MJ Lasers", Fusion Sci and Tech. 56377 (2009)

[14] A. M. Dunne, “A High Power Laser Fusion Facility for Europe”, Nature Physics 2 $2(2006)$

[15] E. I. Moses, et al., "A Sustainable Nuclear Fuel Cycle Based on Laser Inertial Fusion Energy”, Fusion Science and Technology, 56, No. 2, 547-565 (2009)

[16] A. Nikroo, J. Bousquet, R. Cook, B.W. McQuillan, R. Paguio, M. Takagi, "Progress in $2 \mathrm{~mm}$ Glow Discharge Polymer Mandrel Development for NIF," Fus. Sci. 
Technol. 45, 165 (2004)

[17] S.P. Obenschain et al., "Effects of thin high-Z layers on the hydrodynamics of laser-accelerated plastic targets" ,", Phys. Plasmas, 92234 (2002)

[18] R. S. Craxton,F. J. Marshall, M. J. Bonino, R. Epstein, et al., "Polar direct drive: Proof-of-principle experiments on OMEGA and prospects for ignition on the National Ignition Facility", Phys. Plasmas 12056304 (2005)

[19] D. Meyerhofer et al, NIF Direct Drive Program Plan - (next version due early 2010)

[20] A.M. Cok, R. S. Craxton, P.W. McKenty, "Polar-drive Designs for Optimizing Neutron Yields on the National Ignition Facility", Phys. Plasmas 15082705 (2008)

[21] P.W. McKenty, Laboratory for Laser Energetics, University of Rochester, Rochester NY, private communication (2009)

[22] M. Murakami, K. Nishihara, H. Azechi, J. Appl. Phys. 74802 (1993)

[23] M. Murakami, Fusion Eng Design 44111 (1999)

[24] M. Murakami et al. submitted to Phys Plasmas (2010)

[25] DAKOTA - Design Analysis Kit for Optimization and Terascale Applications, Sandia National Laboratory, Albuquerque NM, http://www.cs.sandia.gov/DAKOTA/index.html

[26] S.E. Bodner, et al, "Direct Drive Laser Fusion: Status and Prospects", Phys. Plasmas, 51901 (1998)

[27] J.E. Rothenberg, J. Opt. Soc. Am. B 14, 1664 (1997).

[28] J.A. Marozas, et al. "An Alternative Laser-Speckle-Smoothing Scheme for NIF", Bull. Amer. Physical Soc, 53 (2008)

[29] R. Betti , et al. J. Phys.: Conf. Ser. 112022024 (2008)

[30] W. Theobald, R. Betti, V.A. Smalyuk, K.S. Anderson, et al, "Shock Ignition Experiments on OMEGA at NIF Relevant Intensities, Phys. Rev. Lett. To be published (2009)

[31] A.Bell, University of Oxford, Oxford UK, private communication (2009)

[32] R. J. Kingham et al, "Kinetics for Fusion: Simulation and modeling of absorption and kinetic electron transport for Shock Ignition (KiTSI)", Proposal (2009)

[33] M. Shaw, W. Williams, R. House, C. Haynam, "Laser performance operations model", Optical Engineering, 43, 2885 (2004) 